\title{
REGISTRO DA ATIVIDADE DO SISTEMA NERVOSO CENTRAL DE UCIDES CORDATUS (BRACHYURA, UCIDIDAE): UM MÉTODO ALTERNATIVO PARA DETECÇÃO DE EFEITOS DE FÁRMACOS E POLUENTES AMBIENTAIS
}

\author{
Amado, L.L. ${ }^{1,}$; Mello, V.J. ${ }^{2}$ \& Hamoy, M. ${ }^{2}$ \\ ${ }^{1}$ Universidade Federal do Pará (UFPA), Campus Belém, Laboratório de Ecotoxicologia (ICB) e Laboratório de \\ Pesquisas e Monitoramento Ambiental Marinho (Instituto de Geociências-IG). \\ ${ }^{2}$ Universidade Federal do Pará (UFPA), Campus Belém, Laboratório de Farmacologia e Toxicologia de Produtos \\ naturais, Instituto de Ciências Biológicas (ICB). \\ *Autor correspondente: Iilian.amado@gmail.com
}

O objetivo deste estudo foi fabricar um eletrodo para obtenção de registros eletroencefalográficos no caranguejo Ucides cordatus, testando eficiência do método com os químicos Pentilenotetrazol (PTZ, convulsivante) e Diazepam (BDZ, anticonvulsivante). Os eletrodos foram confeccionados com agulha em aço inox ultra-fina $(25 \times 0,3 \mathrm{~mm})$. Os eletrodos de referência e de registro foram conjugados a uma distância de $0,3 \mathrm{~mm}$ com acrílico odontológico autopolimerizável. Foram posicionados no pedúnculo ocular direito a uma profundidade de 20 mm. A seguir, os animais foram levados para uma gaiola de Faraday de metal ecrã para a aquisição de registro (10 min), e o conector foi acoplado ao amplificador de alta impedância ajustado com filtragem 0,3 Hz e 300 Hz e amplificação de 2000X, monitorado por um osciloscópio e continuamente digitalizados em uma taxa de 1 $\mathrm{KHz}$ por um computador equipado com uma placa de aquisição de dados. Os dados foram processados através de um software especializado. Os caranguejos $(n=36 ; 230 \pm 28 \mathrm{~g})$ foram divididos em 4 grupos experimentais. O grupo 1 (controle) recebeu solução fisiológica $0,9 \%$ no volume equivalente aos grupos que receberam o PTZ. Os grupos 2 e 3 receberam PTZ (120mg/kg) por via intraocular (PTZ IO) e por via intra-articular (PTZ IA), respectivamente. O grupo 4 recebeu BDZ (BDZ IO, $10 \mathrm{mg} / \mathrm{kg}) 5$ minutos após a aplicação de PTZ, por via intraocular. A análise dos resultados foi feita com base nas diferenças observadas nos espectrogramas e na latência da alteração de comportamento (diminuição da motilidade, excitabilidade sem perda do reflexo de postura, tremor generalizado com perda do reflexo de postura). Os resultados foram comparados por ANOVA, seguida de Tukey $(p<0,05)$. As alterações comportamentais após a administração do PTZ foram influenciadas pela via de aplicação do fármaco, sendo a via IO a de menor latência. O registro da atividade do SNC durante o estado basal no grupo controle revelou a potência de $0,1186 \pm 0,08605 \mathrm{mV}^{2} / \mathrm{Hz} .10^{-3}$. Durante o contato com PTZ por via $\mathrm{IO}$ e via IA ocorreram incrementos estatisticamente significativos nos traçados eletrofisiológicos (PTZ $\mathrm{IO}=1,838 \pm 0,1802 \mathrm{mV}^{2} / \mathrm{Hz} .10^{-3}$ para PTZ IO e PTZ IA =1,652 $\left.\pm 0,3586 \mathrm{mV}^{2} / \mathrm{Hz} .10^{-3}\right)$ em relação ao nível basal. Após a utilização do BDZ a potência registrada apresentou média de $0,3856 \pm 0,1150 \mathrm{mV}^{2} / \mathrm{Hz}^{-10^{-3}}$, retornando próximo ao estado basal. Os resultados indicam a eficiência do eletrodo desenvolvido e a potencialidade da técnica para estudos de efeitos de químicos de ação no SNC.

Palavras-chave: eletroencefalograma, caranguejo uçá, efeitos neurotóxicos, sistema nervoso central, modelo biológico alternativo. 